\title{
The Research about No-vibration Data Storage of Micro-scale and Long-term Ocean Turbulence Measurement
}

\author{
LUAN Xin ${ }^{1}$, XUE Bing ${ }^{1}$, SUN Feng-mei ${ }^{1}$ \\ College of Information Science and Technology \\ Ocean University of China \\ Qingdao, China \\ Email: xuebingsucceed@126.com
}

\author{
YANQi-zhi ${ }^{2}$,SONG Da-lei ${ }^{2}$ (Corresponding author) \\ College of Engineering \\ Ocean University of China, \\ Qingdao, China \\ Email: songdalei@ouc.edu.cn
}

\begin{abstract}
Turbulence played an important role in the evolution of the seawater energy and exchange. Multi-scale, long-term, fixed-point and continuous sampling is a new research direction in the turbulence observation. This dissertation designed high-capacity and no-vibration data storage solutions aiming at long-term, continuous turbulence observations. First a multi-scale submerged buoy observing platform is designed. Base on the turbulence observing platform, a multi-parameter data acquisition and no vibrations storage system is designed. This paper describes the hardware and software design implementation of large-capacity data storage arrays in details as well as the readability and easy operation of the transplant of FatFS. Actual test and sea trial prove the design can be achieved large-capacity data access of long-term observation of ocean turbulence base on the submerged buoy.
\end{abstract}

Keywords- Micro-scale turbulence;Long-term observation; SD storage array; FatFS

\section{INTRODUCTION}

The energy transfer process of oceanic internal generally from the large-scale to small scale, finally in the form of turbulent mixing. Turbulent energy dissipation process has great significance for the establishment perfect physical model and the study of marine internal movement ${ }^{[1]}$. The development of ocean turbulence research is closely related to the research and development of observation platforms and observation instruments. Multi-scale, long-term, fixedpoint and continuous sampling is a new research direction in the turbulence observation. The long-term observation certainly will bring a lot of data. The data storage of traditional ocean observing instrument general uses a flash chip soldered directly on the circuit. The flash chip is lower capacity, high cost and cannot be reused. In addition the data exported from the flash chip need to spend a lot of time, and does not support breakpoints transmission. Currently the pluggable storage devices popular on the market are U disk, mobile hard disk, SD card, etc. U disk requires additional drive circuit to achieve communication with processor. Mobile hard disk has large capacity, but during the work process disk movement vibration noise had a greater influence on the turbulence data. SD card has many advantages such as durable, reliable, safe, large capacity, small size, good compatibility, low noise, and low power consumption and so on, so it is very suitable for long-term turbulence data storage ${ }^{[2]}$. Taking into account that turbulence observed data acquisition has high frequency characteristics, the capacity of a separate SD card is difficult to meet the needs of long-term observations. This article designs SD cards storage arrays to solve the problem of marine high-capacity data storage.

\section{MULTI-SCALE AND LONG-TERM ANCHORED OBSERVATION PLATFORM}

Marine submerged buoy system as a way to get the information of the marine environment, which has some advantages such as long observation time, good for hiding and less affected by sea conditions so it is paid more attention by marine scientists. Generally speaking submerged buoy system is made up of gravity anchor, acoustic release, cables, observation instruments and floating body. Depending on the different observation purposes, the submerged buoy system equipped with different instruments. The correct deployment of submerged buoy is the premise of complete, safe recovery. Marine submerged buoy system generally deployed by the research vessel and the observation instruments (whose parameters are set on the shore) underwater observe for long-period and save the observational data automatically. Currently the real-time transmission of submerged buoy can realizes real-time transmission of the data. After the predetermined time, research vessel reaches the original position, deck unit sends instruction and then the acoustic releaser receives instruction and releases the gravity anchor block. At last the whole system floats and instruments are recovered. Multi-scale ocean submersible buoy observation platform shown in figure 1 is composed of five units. The first unit is CTD, mainly used to measure temperature, conductivity, instrument depth; The second unit is self-contained turbulence observation instrument measuring micro-scale turbulence; The third unit is the current meter used to measure the flow rate to study the large-scale circulation; The fourth unit is acoustic release used for the recovery of the instrument; The final unit is gravity anchor which can provide the gravity of the entire system. The research and development of self-contained turbulence observation instrument is independent and a detailed description of the storage system is followed. 


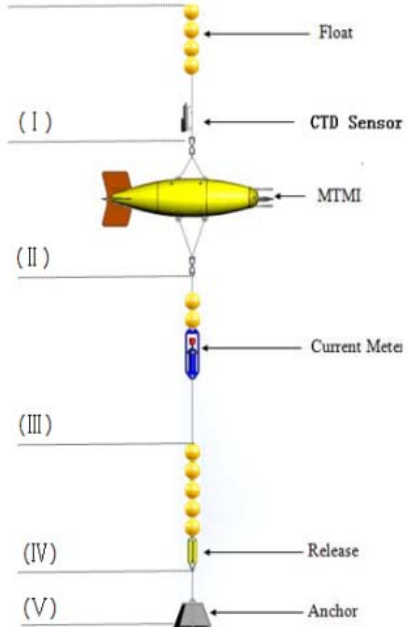

Figure 1 Multi-scale Ocean submerged buoy observation platform

\section{MULTI-PARAMETER DATA ACQUISITION AND STORAGE SYSTEM DESIGN}

Taking into account the observations principle of shear probe, to make the sensitive axis of the shear probe mutually perpendicular as well as make mutual comparison between the data, the turbulence observation instrument installed two shear probes measured simultaneously. The attitude sensor records attitude angle (heading, pitch, and roll) and the accelerations signal on $\mathrm{X}$-axis, $\mathrm{Y}$-axis and $\mathrm{Z}$-axis of the instrument in the water. Data processing using motion compensation correction technique corrects the shear signal power spectrum. Turbulent signal data acquisition and storage block diagram is shown in the figure2.

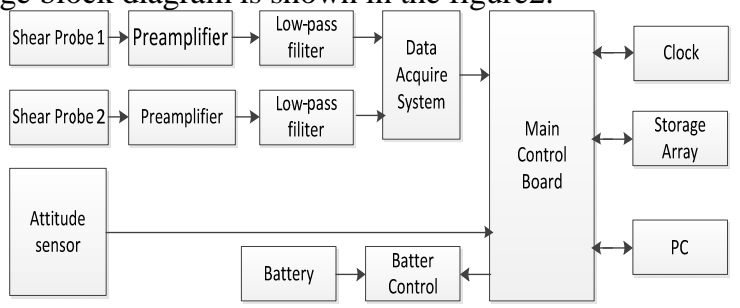

Figure 2 turbulence observation instrument circuit system

Shear probe force and generate signal, output digitals after high-pass filter, voltage amplifier and low-pass filter. Digital is sent to control board through serial port. Control board select the data flow base on the system operating mode (real-time or self-contained mode).Under real-time mode, data is sent directly to the PC and under the self-contained mode data stored in the SD card of storage array. The main control board communicates with PC via the serial port. Through the serial software the system time, the system start time, end time, and the system working mode as well as the work mode or status can be set or detected.

\section{High-CAPACITY DATA STORAGE RESEARCH}

Taking into account the acquisition frequency of ocean turbulence is high, measure up $1024 \mathrm{~Hz}$, acquisition time is long, up to half of a year or more, which depends on the research work plan, Sampling multi-parameter (Attitude, Acceleration, and Shear), so designed large-capacity data storage and following will describe in detail.

\section{A. Hardware Design of Data Storage Array}

SD card support two operating modes, they are serial mode (SPI mode) and parallel mode (SD mode). The advantage of the SPI mode is that it not only contains very good hardware interface with micro controller but also simple than SD mode.SD card has 9 pin, four of them is data port. In our design we choose the Micro-SD card as the better choice, because considering the size and instrument space. The clock frequency of SD card can reach $25 \mathrm{MHZ}$ by default, but in high speed mode it can arrive 50MHZ. The operation voltage of SD card can be $2.7 \mathrm{~V}$ to $3.6 \mathrm{~V}$ and the capacity can be selected from $4 \mathrm{MB}$ to $32 \mathrm{~GB}$ at present ${ }^{[3] \text {. }}$

In SPI mode the definition of each pin shown below:

TABLE I. SD CARD PIN DEFINITION IN SPI MODE

\begin{tabular}{|l|l|l|l|l|l|}
\hline Pin & Name & $\begin{array}{l}\text { Descripti } \\
\text { on }\end{array}$ & Pin & Name & $\begin{array}{l}\text { Descri } \\
\text { ption }\end{array}$ \\
\hline 1 & CS & $\begin{array}{l}\text { Chip } \\
\text { select }\end{array}$ & 6 & VSS & Ground \\
\hline 2 & MOSI & $\begin{array}{l}\text { Master } \\
\text { Out } \\
\text { Slave In }\end{array}$ & 7 & MISO & $\begin{array}{l}\text { Mater } \\
\text { IN } \\
\text { Slave } \\
\text { Out }\end{array}$ \\
\hline 3 & VSS & Ground & 8 & RSV & \\
\hline 4 & VDD & $\begin{array}{l}\text { Power } \\
\text { Supply }\end{array}$ & 9 & RSV & \\
\hline 5 & CLK & $\begin{array}{l}\text { External } \\
\text { Clock }\end{array}$ & & & \\
\hline
\end{tabular}

SD card communicate with micro controller through the four lines in the SPI mode, they are CS、CLK、MISO、 MOSI. SPI is a full-duplex synchronous serial interface, supporting master modes, slave modes, and multi-master environments. Considering the characteristics of SPI mode, the storage uses 12 piece of SD cards connection by sharing channel. Each CLK, MISO, MOSI, CS of the SD card respectively connected to different IO port of the CPLD. The control lines of main control board connect to the CPLD to control the connection of SPI bus with SD card in different time. CPLD only work as a switch.SD card storage system (SPI mode) bus topology structure shown as below figure3. The maximum capacity of each SD card is $32 \mathrm{~GB}$, however the storage array can support maximum capacity of 384 GB.

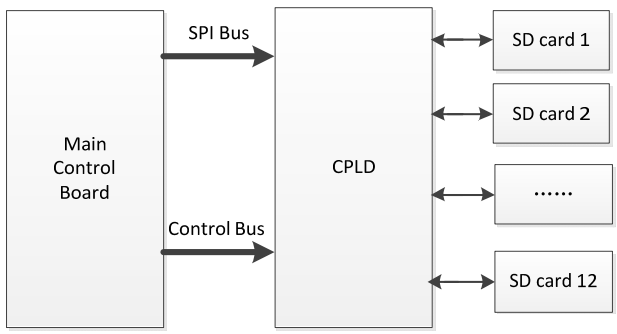

Figure 3 hardware design of data storage array 


\section{B. Software interface design}

1) SD card protocol and initialization

Before using the SD card we should initialize them. After successful initialization, the SD card can be read and written by sending the appropriate commands. In recent years, with the emergence of large-capacity SD card, The original SD1.x protocol cannot meet the requirements of large-capacity SDHC(Secure Digital High Capacity), so SD card protocol standards have been upgraded to SD2.0.Many electronic products cannot drive the large-capacity SD card, it need give full consideration to the type of SD card when drive SD card.SD card protocol include SD1.X and SD2.0.SD2.0 include standard-capacity SD card protocol and large capacity SDHC protocol. SDHC or high-capacity SD memory card refers to a capacity greater than 2GB less than or equal to 32GB SD card, but SDHC can only support the FAT32 file system $^{[4]}$. The biggest difference between SD1.x and SD2.0 is that the command address, the SD2.0 address unit is block, SD1.x is bytes.SD card initialization process as shown below.

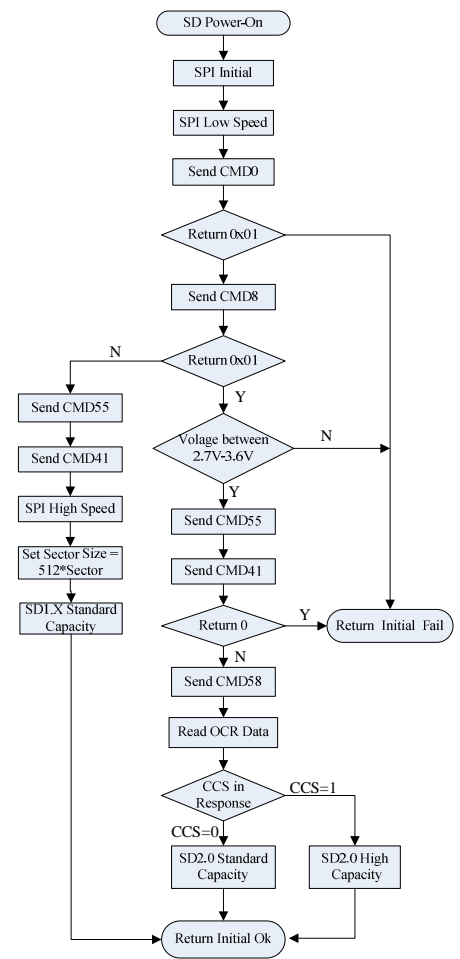

Figure 4 process of SD card initialization

In the SD card initialization process, Firstly, send CMD0 command to enters idle mode, then send CMD8 command to determine if the card is SD2.0.If the card is SD2.0, it will return 0x01,otherwise it will return 0x05,according to the above operation the SD type is determined. Different operating on the different card, the SD card initialization process is completed.

2) SD cards traversal processes

In this design, using $12 \mathrm{SD}$ cards to share channel in different time, so how to use the SD card reasonably is the key to the system design. In the process of writing data to the $\mathrm{SD}$, it needs to determine whether SD card capacity is greater than a set value (SD card reserved capacity),if it meets the conditions, the SD card can be read and written, otherwise, the SPI bus should be connected to the next SD card.

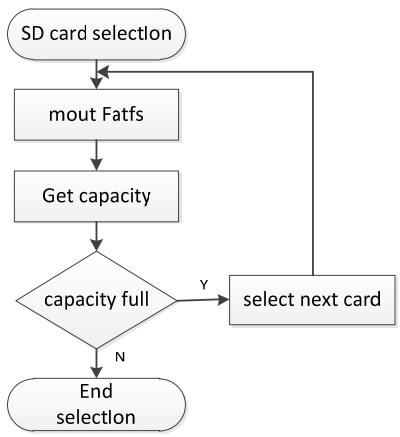

Figure 5 process of SD card traversal

\section{THE IMPLEMENTATION OF FATFS}

The file system is the core to manage SD data. On the one hand, it connects the operating system on the upper layer and completes the instruction issued by the operating system. On the other hand, it manages the lower SD memory card and provides a real-time operation of the bridge between the operating system and SD memory card. Considering the convenience of operate and the portability of the code of the file system, the design transplant FatFS ${ }^{[2]}$. FatFS that is a generic file system module provide FAT file system for small embedded systems. The development of FatFS follow ANSI C, therefore it does not depend on the hardware platform. FatFS structure is shown in the figure 6 below.

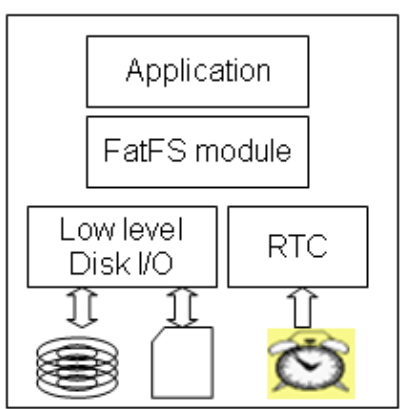

Figure 6 FatFS structure

Because the FatFS module is completely separated from the disk I/O layer so it requires five functions to achieve read and write as well as access the current time function of the underlying physical disk.

- disk_initialize(),initialize the disk drive

- disk_read(),read sectors

- disk_write(),write sector

- disk_ioctl(),control the properties of the related SD

- get_fattime(),get the current time

The first three functions are the necessary operation of transplantate file system and the latter two functions the user 
can choose according to their needs. They mainly consists of the SD card initialization function SD_Init (), the function of read the single block SD_ReadSingleBlock (), the function of write a single block SD_WriteSingleBlock ().The three functions is the key of the SD card driver. After the file system transplantation is completed its interface functions can be called. Mainly used functions are shown in the following table. Note that when switch to a SD card, f_mount () function should be recalled otherwise it will not be able to read and write to the SD card.

TABLE II. SOME FUNCTIONS USE IN THE DESIGN

\begin{tabular}{|l|l|l|}
\hline Number & \multicolumn{1}{|c|}{ Name } & \multicolumn{1}{c|}{ Main function } \\
\hline 1 & f_mount & Register/Unregister a work area \\
\hline 2 & f_open & Open/Create a file \\
\hline 3 & f_close & Close a file \\
\hline 4 & f_write & Write file \\
\hline 5 & f_putc & Write a character \\
\hline 6 & f_getfree & Get free clusters \\
\hline
\end{tabular}

VI. TRIAL EXPERIMENT ANALYSES

\section{A. Long-term stability test}

January 2012, the entire data acquisition and storage system was installed in a test submerged buoy system. The system was put into a pool of still water for long-term storage system reliability test. When the entire system was recovered in July, large-capacity storage array works stably and the storage data are complete and reliable.

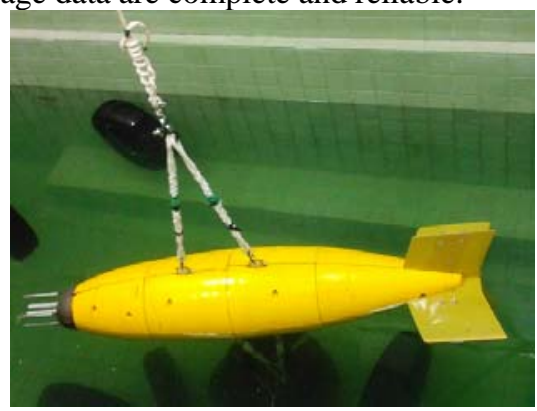

Figure 7 the picture of long time test

\section{B. Data Correctness Test}

During the South China Sea test in September 2012, the turbulence data acquisition and storage system was integrated in the submerged buoy. The turbulent kinetic energy dissipation rate is shown in the following figure.

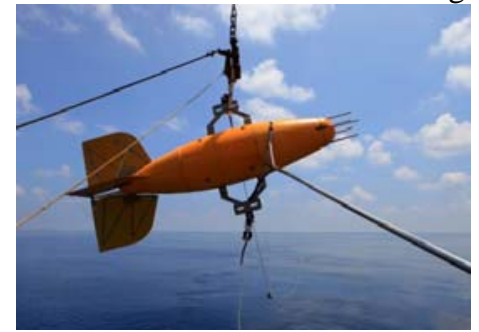

Figure 8 the Deployment of turbulence instrument

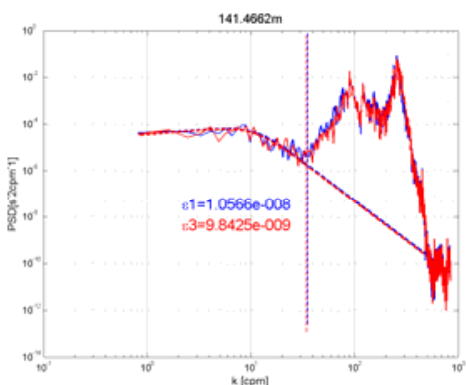

Figure 1. the comparison between theoretical spectrum and actual spectrum

It can be seen through the figure that the number of dissipation rate the two probes calculated is same and the two probes are in good agreement and the actual spectrum coincide well with the theoretical spectrum.

\section{CONCLUSIONS}

In this paper, aiming at the problem ocean long-term, fixed-point, multi-parameter observation generates a large amount of data, a high-capacity data storage solution up to 384GB is designed. And then hardware and software design of the turbulence instrument is started. The dissertation introduces the solution of 12 SD cards shared channel in different time. Long-term pool experiments prove that this design is reasonable and stability, sea trial results says that turbulence instrument used in marine submerged buoy system is correctly.

\section{REFERENCES}

[1] Rolf Lueck and Daniel Huang,"Dissipation Measurement with a Moored Instrument in a Swift Tidal Channel," J, American Meteorological Society, 1999, pp1499-1505.

[2] FatFs Generic FAT File System Module http://elm-chan.org/ fsw/ff/00index_e.html

[3] SanDisk Secure Digital Card Product Manual

[4] Version 1.9 Document No. 80-13-00169 December 2003

[5] Interfacing SD Cards with Blackfin ${ }^{\circledR}$ Processors Contributed by Aseem Vasudev and Jay anti Addepalli Rev 1 - February 19, 2010

[6] Oakey, N.S, "An instrument to measure oceanic turbulence and microstructure," Bedford Inst. Oceanogr. Rept. Ser.BI-R-77-3, Dartmouth, N.S. Canada, 1997, 52pp.

[7] Rolf Lueck "Converting shear probe, thermistors and microconductivity signals into physical units ”,Rockland Science International Inc ,2010,pp1-6

[8] Osborn, T. R. and W. R. Crawford, 1980: An airfoil probe for measuring turbulent velocity fluctuations in water. Air-Sea Interaction: Instruments and Methods, F. W. Dobson and R. Davis, Eds., Plenum, 369-386 